\title{
User profiling with geo-located posts and demographic data
}

\author{
Adam Poulston, Mark Stevenson and Kalina Bontcheva \\ Department of Computer Science \\ University of Sheffield \\ Sheffield S1 4DP, UK \\ \{ARSPoulston1, k.bontcheva, mark.stevenson\}esheffield.ac.uk
}

\begin{abstract}
This paper presents a novel method for user profiling in social media that makes use of geo-location information associated with social media posts to avoid the need for selfreported data. These posts are combined with two publicly available sources of demographic information to automatically create data sets in which posts are labelled with socio-economic status. The data sets are linked by identifying each user's 'home location'. Analysis indicates that the nature of the demographic information is an important factor in performance of this approach.
\end{abstract}

\section{Introduction}

Previous research has shown that the language a person uses on-line can be indicative of a wide range of personal characteristics, including gender, age (Schler et al., 2006), personality (Schwartz et al., 2013), political ideology (Sylwester and Purver, 2015) and occupational class (Preotiuc-Pietro et al., 2015a). Several user profiling models that predict these characteristics have been developed, some of which have accuracy that exceeds human performance (Burger et al., 2011; Youyou et al., 2015). User profiling models have applications such as gendered behaviour analysis (Purohit et al., 2015) and bias reduction in predictive models (Culotta, 2014).

Previous work on user profiling has traditionally relied on profiles annotated with self-reported characteristics for training data. This can be difficult to acquire in large quantities and forms a bottleneck in the development of user profiling systems.
Recently, approaches have attempted to build user profiling datasets through other means. PreoţiucPietro et al. (2015a)(2015b) extracted known job titles from Twitter profile descriptions to annotate users with occupational class and income. Crowdsourcing techniques have been used to annotate data-in Kosinski et al. (2013) users were invited to complete a personality quiz and then asked if they wanted to share their data for research purposes. In a similar fashion, Nguyen et al. (2014) provided an application which attempts to guess user's age and gender based on their Twitter profile and then asks for the correct answer afterwards. Profile information has also been collected from websites; such as blogs (Schler et al., 2006; Burger et al., 2011) or review sites (Hovy et al., 2015).

Many countries regularly conduct surveys of their population that provide large-scale demographic information, some of which is made freely available. For example, the United Kingdom conducts a mandatory census every decade. Although the full information collected is not made publicly available for a century, aggregated information is made freely available. In addition, governments are increasingly making data available for research purposes, some of which may be relevant to user profiling ${ }^{1}$. This data has the advantage of providing population-level information gathered using reliable methods.

This paper explores how demographic information can be used to assist with the development of user profiling models. It describes an approach to the generation of annotated training data by combining geo-located social media profiles with geo-

\footnotetext{
'e.g. http: //data.gov.uk/
} 
graphically linked demographic data. This approach is applied using publicly available demographic data describing socio-economic status (Section 2). A set of geo-located tweets is collected and the 'home location' of each user identified using a clustering approach so that each profile can be mapped onto the regions in the demographic data, thereby providing labelled data (Section 4) which is used to create a user profiling system (Section 5).

\section{Data Sources}

This work makes use of data from two sources: demographic data provided by the UK Office for National Statistics demographic data and Twitter posts.

Demographic data provides information about characteristics of a population within a specified area. The UK government provides open datasets containing information about a range of demographic variables including highest qualification, job category and unemployment rates.

This paper makes use of geodemographic segmentation datasets in which an area, or individual's, demographics are generalised into a single socio-economic category. These types of data sets are often used for marketing purposes (Troy, 2008). The United Kingdom's Office for National Statistics $(\mathrm{ONS})^{2}$ provides a range of data sets including the Output Area Classification (OAC) and Local Authority Classification (LAC) datasets. Unlike commercial datasets, such as MOSAIC ${ }^{3}$ and Acorn ${ }^{4}$, the methodology used to develop the OAC and LAC datasets is fully documented.

The OAC data set is organised around output areas, regions of around 200 households in England and Wales. The OAC dataset places residents of every Output Area into a hierarchy of socio-economic groups based on responses to the 2011 UK Census. The dataset consists of a hierarchical classification scheme with three layers: supergroups (shown in Figure 1), groups and subgroups. For example, the Output Area E00124315 is associated with the '7-constrained city dwellers' supergroup, the '7achallenged diversity' group, and the '7a2-hampered aspiration' subgroup.

\footnotetext{
${ }^{2}$ http://www.ons.gov.uk

${ }^{3}$ http://www.experian.co.uk/mosaic/

${ }^{4}$ http: //acorn.caci.co.uk/
}

1 Rural Residents

2 Cosmopolitans

3 Ethnicity Central

4 Multicultural Metropolitans

5 Urbanites

6 Suburbanites

7 Constrained City Dwellers

8 Hard-Pressed Living

Figure 1: OAC supergroups

1 English and Welsh Countryside

2 Scottish and Northern Irish Countryside

3 London Cosmopolitan

4 Suburban Traits

5 Business and Education Centres

6 Coast and Heritage

7 Prosperous England

8 Mining Heritage and Manufacturing

Figure 2: LAC supergroups

The LAC dataset is organised in a similar way to the OAC dataset, with eight supergroups (shown in Figure 2) and two layers of subgroups, but is generalized to cover Local Authorities (also provided by the UK Data service describing areas governed by a single council covering the whole of the UK), which are larger than Output Areas. Despite some similarities in names, the two datasets use different classification strategies leading to categories not being directly comparable.

Geo-located social media posts from the United Kingdom were identified using the Twitter public streaming API. The Twitter REST API was then used to retrospectively collect each user's tweets (up to 3200 per user) and any public information on their profile. Users with fewer than 50 geo-located tweets were excluded to ensure sufficient data was available for subsequent processing. Just over 135,000 profiles were initially collected, 86,262 of which had enough tweets. A small portion of profiles $(3,743)$ not representative of the general population (e.g. profiles of celebrities, shops, spammers) were excluded using standard approaches (Chu et al., 2012; Cresci et al., 2015), leaving 82,519 profiles used for experiments described later. 


\section{Home location identification}

Demographic data provides information about individuals based on their residential address, making it important to make sure that a user is associated with that location rather than where they happened to be when sending a particular tweet. Consequently all users in the dataset were assigned a 'home location' in the form of a latitude-longitude coordinate.

Our approach assumes that each social media user commonly posts from a limited set of locations. It is further assumed that the location posted from the most often is the user's home location. The task of assigning 'home location' given a collection of geo-located posts is then approached as a clustering problem based on geo-location information associated with their tweets. Other approaches for assigning home location were considered, such as as those that consider textual (Han et al., 2014) and social network (Jurgens et al., 2015) cues, but these typically only produce accurate judgements at the city level, whereas demographic datasets often operate at a finer scale.

The coordinates of each user's geo-located posts are clustered using k-means, with $\mathrm{k}$ set using the 'jump' method (Sugar and James, 2011). (A range of alternative clustering algorithms were also explored but were not found to significantly improve performance.) The most populous cluster was then identified and the point closest to the cluster centroid taken as the home location. Cluster 'density' was calculated; defined as the average Vincenty distance (Vincenty, 1975) (a model of geographic distance) in miles between each data point and the cluster centroid. This provides the option to exclude users with highly uncertain home location (i.e low density home cluster).

\subsection{Evaluating Home Location Assignment}

Our method for home location identification was assessed by comparing self-reported locations from the 'location' field with those assigned by clustering. Only 728 of the 82,519 profiles include a selfreported location. Of these, 176 were discarded as being clearly fictitious; leaving 552 profiles for evaluation. These were further cleaned by manually removing extraneous information such as emoticons.

Varying levels of granularity were present in the declared location fields, ranging from street level to country, with the majority at town or city level, e.g. 'Edinburgh'. A number of the location fields also included a single coordinate location. The Nominatim geocoding tool ${ }^{5}$ was used to convert the selfreported locations to geographical coordinates. Vincenty distance between these coordinates and the assigned home location was calculated.

The majority of distances $(69.7 \%)$ were accurate to within 10 miles, more than half $(56.9 \%)$ accurate to within 5 miles and $30.8 \%$ within 1 mile. The home location gained from the location text field is only expected to be accurate to within 5 or 10 miles because the majority of self-reported locations are towns or cities. The results given here therefore suggest that the clustering approach presented here can identify the home location of a Twitter user with reasonable accuracy.

\section{Demographically Labelling Data}

A data set was created in which each social media profiles were associated with their corresponding OAC and LAC supergroup. A home location was assigned to each of the 82,519 profiles identified of Section 2 using the approach described in Section 3. Point-in-polygon tests then linked each profile with its relevant Output Area and Local Authority. Once a profile was allocated an associated boundary, demographic linking is a simple look-up task.

Two user profiling datasets were created by linking users with their local demographics; users in England and Wales were labelled with one of eight OAC supergroups associated with that user's local Output Area, and users across the whole of the UK were labelled with one of eight LAC supergroups associated with their Local Authority. These datasets are referred to as 'OAC-P and 'LAC-P', respectively.

\section{User demographic prediction}

We approach our analysis as a classification problem, aiming to use the content of a user's tweets to predict their LAC-P and OAC-P from the eight possible classifications in each data set.

A classification pipeline was created, taking each user's corpus of tweets as input, tokenized using a

\footnotetext{
${ }^{5}$ http://openstreetmap.org/
} 
Twitter aware tokenizer (Gimpel et al., 2011). TFIDF transformed word n-grams (1- and 2-grams) were used as features for a multi-class Support Vector Machine (SVM) with a linear kernel. n-grams and SVMs were chosen as they have been shown to consistently perform well at user profiling tasks, both for social media (Rao and Yarowsky, 2010; Rout et al., 2013; Schwartz et al., 2013) and other types of text (Boulis and Ostendorf, 2005; Garera and Yarowsky, 2009), and are as such a useful tool to establish baseline performance. Balanced sets were extracted from the OAC-P and LAC-P datasets with 2000 members per label in both cases. 10-fold crossvalidation was used for all experiments.

\subsection{Results}

The results of the SVM classifier are presented in Table 1, compared with results from a random baseline. Prediction of both OAC and LAC outperform the random baseline, indicating that the training dataset described in this article can be used to create valuable user profiling systems. Results for LAC are encouraging and indicate that it is possible to achieve promising results while using a simple classifier. The OAC predictions are noticeably worse than LAC but still outperform the baseline.

The large difference in performance obtained using the two data sets may be down to differences in their nature. Analysis revealed that the regions defined in the OAC-P dataset are smaller than those in the LAC-P dataset; the average length of the diagonal of the minimum bounding rectangle for each region is 0.93 miles for Output Areas, whereas it is 34.5 miles for Local Authorities. It seems probable that profiles are more likely to be mis-classified when assigned to more fine-grained regions in the OAC-P data set, resulting in a noisier data set.

Another difference between the data sets is that the OAC scheme aims to model 'geographically independent socio-economic status' in contrast to the LAC categories which are region dependent (e.g. 'London Cosmopolitan'). Examination of the highest ranked features by SVM coefficient for each LAC supergroup revealed a connection between groups and geography. The most important features for many classes are words or phrases referencing specific areas in the UK as well as several stereotypical dialect features. For example, the '3-

\begin{tabular}{|l|l|l|}
\hline & OAC-P & LAC-P \\
\hline Random Baseline & 0.1259 & 0.1259 \\
\hline SVM classifier & 0.2757 & 0.5047 \\
\hline
\end{tabular}

Table 1: Accuracy for OAC-P and LAC-P prediction

London Cosmopolitan' supergroup's highest ranked features relate exclusively to London, its surrounding boroughs and public transport system. In contrast, the OAC's feature coefficients are not as location dependent; for example, '1-Rural Residents' contains features such as 'Severn' (a river), 'stables', 'mountain bike' and 'emmerdale' (a UK soap opera set in the countryside). Similarly, "4-Multicultural Metropolitans' is the only group identified that has non-English phrases and the Islamic holidays Eid and Ramadan as important features-a promising sign given the supergroup title.

\section{Conclusion}

This paper explored the use of population-level demographic information for user profiling. It presented a novel approach to the generation of automatically labelled data by making use of geo-located social media posts. The 'home location' for a user is identified using clustering and then combined with publicly available information from two previously unexplored demographic datasets. A simple classifier based solely on tweet content was able to predict socio-economic status with promising results for one data set.

Analysis indicated that the properties of the demographic data are important. Key factors include the granularity of the output area and degree to which the groupings are based on socio-economic, rather than geographic, characteristics rather than geographic features.

The demographic data sets used in this work have the advantages that they are large-scale and collected using sound methodologies. However, the information they contain is aggregated and is updated infrequently. Our future work will explore the extent to which these disadvantages can be overcome. Accurate identification of home location is important for the approach presented here. We will also explore its effect on overall performance and approaches for identifying home location more accurately.

Code available at https://github.com/ adampoulston/geo-user-profiling. 


\section{References}

Constantinos Boulis and Mari Ostendorf. 2005. A quantitative analysis of lexical differences between genders in telephone conversations. In Proceedings of the 43rd Annual Meeting on Association for Computational Linguistics, pages 435-442. Association for Computational Linguistics.

John D Burger, John Henderson, George Kim, and Guido Zarrella. 2011. Discriminating gender on twitter. In Proceedings of the Conference on Empirical Methods in Natural Language Processing, pages 1301-1309. Association for Computational Linguistics.

Zi Chu, Steven Gianvecchio, Haining Wang, and Sushil Jajodia. 2012. Detecting automation of twitter accounts: Are you a human, bot, or cyborg? Dependable and Secure Computing, IEEE Transactions on, 9(6):811-824.

Stefano Cresci, Roberto Di Pietro, Marinella Petrocchi, Angelo Spognardi, and Maurizio Tesconi. 2015. Fame for sale: efficient detection of fake twitter followers. Decision Support Systems, 80:56-71.

Aron Culotta. 2014. Reducing sampling bias in social media data for county health inference. In Joint Statistical Meetings Proceedings.

Nikesh Garera and David Yarowsky. 2009. Modeling latent biographic attributes in conversational genres. In Proceedings of the Joint Conference of the 47th Annual Meeting of the ACL and the 4th International Joint Conference on Natural Language Processing of the AFNLP: Volume 2-Volume 2, pages 710-718. Association for Computational Linguistics.

Kevin Gimpel, Nathan Schneider, Brendan O'Connor, Dipanjan Das, Daniel Mills, Jacob Eisenstein, Michael Heilman, Dani Yogatama, Jeffrey Flanigan, and Noah A Smith. 2011. Part-of-speech tagging for twitter: Annotation, features, and experiments. In Proceedings of the 49th Annual Meeting of the Association for Computational Linguistics: Human Language Technologies: short papers-Volume 2, pages 42-47. Association for Computational Linguistics.

Bo Han, Paul Cook, and Timothy Baldwin. 2014. Textbased twitter user geolocation prediction. Journal of Artificial Intelligence Research, pages 451-500.

Dirk Hovy, Anders Johannsen, and Anders Søgaard. 2015. User review sites as a resource for large-scale sociolinguistic studies. In Proceedings of the 24th International Conference on World Wide Web, pages 452-461. ACM.

David Jurgens, Tyler Finethy, James McCorriston, Yi Tian Xu, and Derek Ruths. 2015. Geolocation prediction in twitter using social networks: A critical analysis and review of current practice. In Proceed- ings of the 9th International AAAI Conference on Weblogs and Social Media (ICWSM).

Michal Kosinski, David Stillwell, and Thore Graepel. 2013. Private traits and attributes are predictable from digital records of human behavior. Proceedings of the National Academy of Sciences, 110(15):5802-5805.

Dong Nguyen, Dolf Trieschnigg, and Theo Meder. 2014. Tweetgenie: Development, evaluation, and lessons learned. COLING 2014, pages 62-66.

Daniel Preoţiuc-Pietro, Vasileios Lampos, and Nikolaos Aletras. 2015a. An analysis of the user occupational class through Twitter content. Proceedings of the 53rd Annual Meeting of the Association for Computational Linguistics and the 7th International Joint Conference on Natural Language Processing (Volume 1: Long Papers), pages 1754-1764.

Daniel Preoţiuc-Pietro, Svitlana Volkova, Vasileios Lampos, Yoram Bachrach, and Nikolaos Aletras. 2015b. Studying user income through language, behaviour and affect in social media. PloS one, 10(9):e0138717.

Hemant Purohit, Tanvi Banerjee, Andrew Hampton, Valerie L Shalin, Nayanesh Bhandutia, and Amit P Sheth. 2015. Gender-based violence in 140 characters or fewer: A\# bigdata case study of twitter. arXiv preprint arXiv:1503.02086.

Delip Rao and David Yarowsky. 2010. Detecting latent user properties in social media. In Proc. of the NIPS MLSN Workshop. Citeseer.

Dominic Rout, Kalina Bontcheva, Daniel PreoţiucPietro, and Trevor Cohn. 2013. Where's@ wally?: a classification approach to geolocating users based on their social ties. In Proceedings of the 24th ACM Conference on Hypertext and Social Media, pages 11-20. ACM.

Jonathan Schler, M Koppel, S Argamon, and J Pennebaker. 2006. Effects of age and gender on blogging. In Proceedings of AAAI Spring Symposium on Computational Approaches for Analyzing Weblogs: Papers from the AAAI Spring Symposium, pages 199-205.

H. Andrew Schwartz, Johannes C. Eichstaedt, Margaret L. Kern, Lukasz Dziurzynski, Stephanie M. Ramones, Megha Agrawal, Achal Shah, Michal Kosinski, David Stillwell, Martin E. P. Seligman, and Lyle H. Ungar. 2013. Personality, gender, and age in the language of social media: The open-vocabulary approach. PLoS ONE, 8(9):e73791, 09.

Catherine A Sugar and Gareth M James. 2011. Finding the number of clusters in a dataset. Journal of the American Statistical Association.

Karolina Sylwester and Matthew Purver. 2015. Twitter language use reflects psychological differences between democrats and republicans. PLOS ONE, 10(9):e0137422, 09. 
Austin Troy. 2008. Geodemographic segmentation. In Encyclopedia of GIS, pages 347-355. Springer US.

T. Vincenty. 1975. Direct and inverse solutions of geodesics on the ellipsoid with application of nested equations. Survey Review, 23(176):88-93.

Wu Youyou, Michal Kosinski, and David Stillwell. 2015. Computer-based personality judgments are more accurate than those made by humans. Proceedings of the National Academy of Sciences, 112(4):1036-1040. 